\title{
Reversible Endoscopic Medial Maxillectomy: Endonasal Approach to Diseases of the Maxillary Sinus
} \author{
Carlos Diogenes Pinheiro Neto ${ }^{7}$ \\ 1 Department of ENT and Skull Base Surgery at the Policlinica de \\ Botafogo, Universidade do Estado do Rio de Janeiro, Rio de Janeiro, \\ RJ, Brazil \\ 2 Department of ENT, Policlinica de Botafogo, Rio de Janeiro, RJ, Brazil \\ ${ }^{3}$ Department of Rhinology and Skull Base Surgery, Policlinica de \\ Botafogo, Rio de Janeiro, RJ, Brazil \\ ${ }^{4}$ São Paulo ENT Center, Hospital Edmundo Vasconcelos, São Paulo, SP, \\ Brazil \\ ${ }^{5}$ Department of ENT and HSN/Skull Base Program, Centro Médico \\ Caracas, Caracas, Venezuela \\ ${ }^{6}$ Department of Otolaryngology, Albany Medical Center, Albany, NY, \\ United States \\ ${ }^{7}$ Department of Otolaryngology and Neurosurgery, Albany Medical \\ Center, Albany, NY, United States
}

Miguel Soares Tepedino ${ }^{10}$ Ana Clara Miotello Ferrão ${ }^{2}$ Hana Caroline Morais Higa ${ }^{3}$ Leonardo Lopes Balsalobre Filho ${ }^{4}$ Enrique Iturriaga ${ }^{5}$ Marcelo Charles Pereira $^{6}$

Address for correspondence Miguel Soares Tepedino, MD, PhD, Policlínica de Botafogo, Avenida Pasteur, 72, Botafogo,

Rio de Janeiro - RJ, Brazil, 22290-240 (e-mail: mstepedino@gmail.com).

Int Arch Otorhinolaryngol 2020;24(2):e247-e252.

\begin{abstract}
Keywords

- maxillary sinus

- maxillary diseases

- nasolacrimal duct

- inferior turbinate

Introduction The endoscopic access has reduced the morbidity associated with external approaches in diseases of the maxillary sinus. A reversible endoscopic medial maxillectomy (REMM) is presented as an alternative for treatment of benign maxillary diseases.

Objective To describe the REMM technique and report four cases of patients with benign maxillary sinus conditions treated through this approach.

Methods The present study was divided into two parts: anatomical and case series. Two cadaveric dissections confirmed the feasibility of the REMM approach. The same technique was performed on four consecutive patients with benign maxillary sinus disease.

Results The cadaveric dissections confirmed wide exposure to the maxillary cavity, preserving the anatomy of the maxillary sinus. In the patient series, one patient presented with an antrochoanal polyp, one had a silent sinus syndrome, one had a chronic maxillary sinusitis secondary to a gunshot, and the last one had an inverted papilloma in the maxillary sinus. In all of the cases, the REMM approach provided excellent access and adequate resection, as well as preservation of the inferior turbinate, nasolacrimal duct, and lateral wall of the nose (including its osteomucosal component). Finally, all of the patients had an uneventful postoperative course.

Conclusion The REMM technique is an excellent surgical approach to benign conditions of the maxillary sinus. It has few limitations and appears to be associated with less morbidity than conventional techniques.
\end{abstract}

received

October 4, 2019

accepted

November 5, 2019

published online

February 27, 2020
DOI https://doi.org/

10.1055/s-0040-1701267.

ISSN 1809-9777.
Copyright $(2020$ by Thieme Revinter

Publicações Ltda, Rio de Janeiro, Brazil
License terms

(ㄷ) (i) $\ominus$ (5) 


\section{Introduction}

The advent of endoscopic sinonasal surgery has revolutionized concepts regarding the surgical anatomy of and approaches to the nasal cavity, the paranasal sinuses, and the skull base. ${ }^{1}$ In conditions of the maxillary sinus, endoscopic access has reduced the morbidity associated with external approaches, such as lateral rhinotomy, the Caldwell-Luc procedure, and facial degloving. ${ }^{2}$

Endoscopic medial maxillectomy (EMM) is a radical procedure that involves removal of the uncinate process, the bulla, the inferior turbinate, the middle turbinate, and the medial wall of the maxilla (including the nasolacrimal duct) to provide access to the maxillary sinus. ${ }^{3}$ Despite having lower morbidity compared with conventional open techniques, sequelae such as atrophic rhinitis, epiphora, and recurrent dacryocystitis may occur due to complete removal of the inferior turbinate and of the nasolacrimal duct.

The present study proposes a transnasal endoscopic approach to the maxillary sinus through a reversible endoscopic medial maxillectomy (REMM) as an alternative for the treatment of benign maxillary diseases. This technique is based on an osteomucosal flap that provides wide exposure to the maxillary cavity, while preserving the anatomy and physiology of the maxillary sinus.

\section{Objective}

To describe the REMM technique and report four cases of patients with benign maxillary sinus conditions treated through this approach.

\section{Materials and Methods}

The present study was divided into two parts: anatomical and case series. Two cadaveric dissections (total of four sides) were completed to confirm the feasibility of the REMM approach.

The REMM technique was systematically applied in four patients who underwent surgery at the ENT service of a private general hospital in Rio de Janeiro, Brazil, from January 2018 to January 2019.

All of the patients presented with benign conditions of the maxillary sinus and were treated exclusively by endoscopic endonasal surgery, performed under general anesthesia.

\section{Equipment}

Dissections and surgeries were performed with instruments used in endoscopic sinus surgery, straight and angled osteotomes, 4-mm endoscopes with $0^{\circ}, 30^{\circ}$, and $45^{\circ}$ Hopkins telescopes (Karl Storz, Culver City, CA, USA) and Fusion ENT Surgical Navigation (Medtronic, Dublin, Ireland).

\section{Cadaveric Dissection}

In the cadaveric study, the dissections performed followed the same steps of the surgical procedure.

The uncinate process was removed, the natural ostium of the maxillary sinus was identified, and a wide maxillary antrostomy was performed, providing good visualization of the posterior wall of the sinus. The maxillary line was identified. A fine-tipped monopolar diathermy pencil was used to make an oblique pre-lacrimal incision, starting $0.5 \mathrm{~cm}$ anterior to the superior portion of the nasolacrimal duct and moving inferiorly so as to draw a tangent line from the head anterior portion of the inferior turbinate to the floor of the nose, at the pyriform aperture (-Fig. 1A). This incision extends posteriorly through the floor of the nose to the posterior portion of the inferior turbinate. Some minor mucosal undermining was performed to expose the frontal process of the maxilla (-Fig. 1B, C). A straight osteotome was used to fracture the maxilla along the line of the original incision (-Fig. 1D). The entire medial wall of the maxilla, including the nasolacrimal duct and the inferior turbinate, were displaced medially ( - Fig. 1E). Even with a 0degree endoscope, ample visualization of the posterior, lateral, and most of the anterior walls of the maxillary sinus were possible. Angled endoscopes $\left(30^{\circ}\right.$ or $45^{\circ}$ ) may be used as needed to provide additional visualization of the anterior wall. The maxillary sinus was fully explored. Once it is disease-free, the medial wall was returned to its original position and secured with two sutures with 3-0 Vicryl (-Fig. 1F).

\section{Procedure}

The REMM procedure was performed under general anesthesia. Cotton pledgets soaked in $2 \%$ lidocaine with epinephrine 1:2000 were applied on the nasal mucosa. In the patients, the surgical procedure performed followed the same steps of the cadaveric dissection.

\section{Postoperative care}

Patients were discharged on oral antibiotics 12 hours after surgery. All were instructed to perform nasal irrigation with $0.9 \%$ saline solution. The nasal splint was removed 1 week postoperatively.

\section{Results}

\section{- Cadaveric dissection:}

A total of two adult human cadaver specimens (four sides) were dissected using the REMM surgical approach.

- Patient series:

\section{Case 1}

In december 2017, a 25-year-old male presented with a 3year history of progressively worsening nasal congestion, discharge, and hyposmia, most prominently on the left.

Nasal endoscopy revealed a left-sided polypoid lesion in the middle meatus, extending into the nasal cavity. Computed tomography (CT) of the paranasal sinuses showed complete opacification of the left maxillary sinus, with enlargement of the ostiomeatal complex, and a mass lesion isodense with soft tissue suggestive of an antrochoanal polyp (-Fig. 2A).

Surgery was performed through an exclusively endoscopic endonasal approach. Initially, a wide maxillary antrostomy was performed, but did not provide visualization of the attachment of the polyp (localized to the lateral and anterior walls of the left maxillary sinus). A left-sided REMM was then 


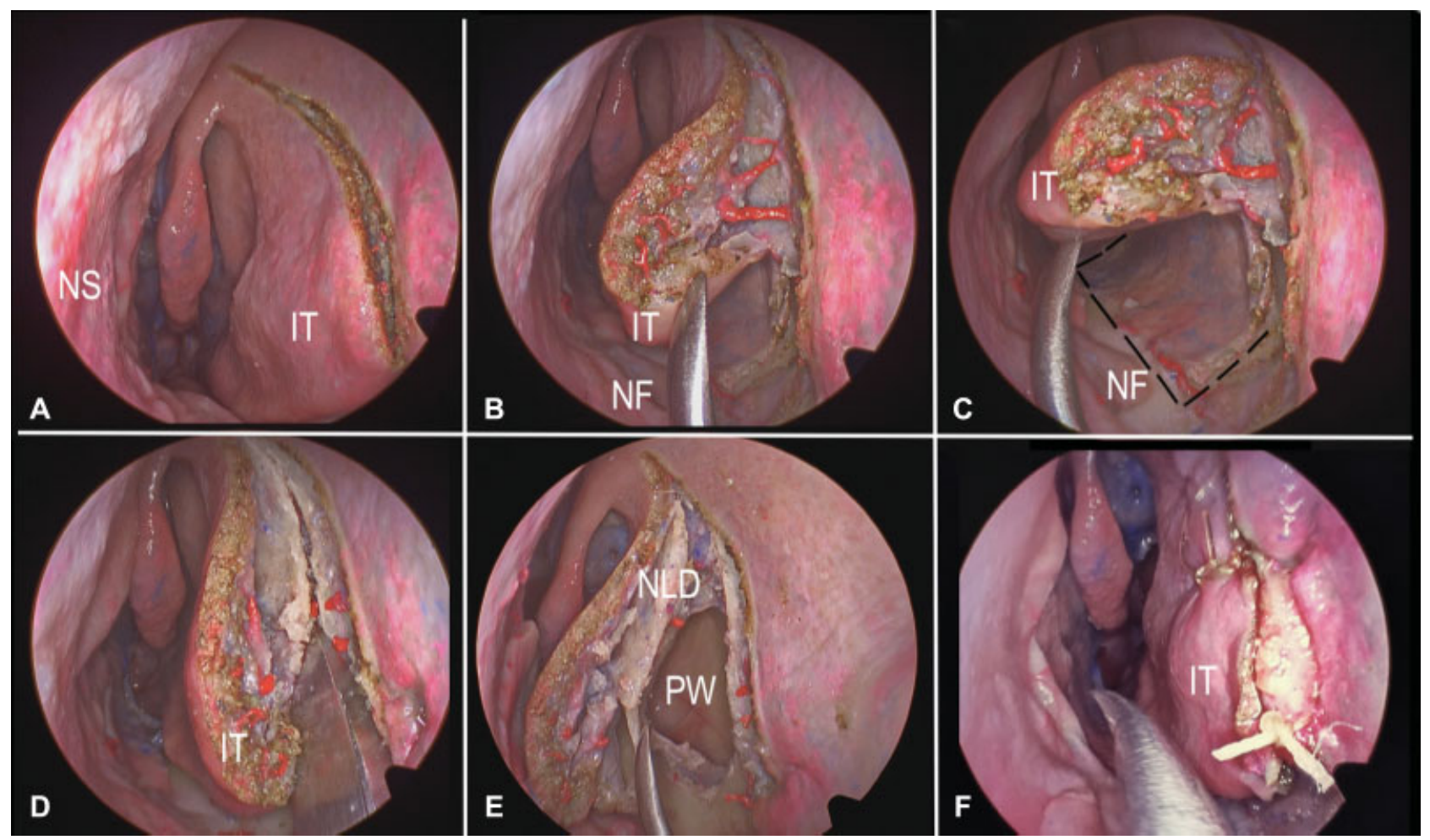

Fig. 1 Endonasal endoscopic cadaveric dissection showing the steps of the REMM on the left side (A-F). Pictures obtained with a 0-degree scope. (A) Oblique pre-lacrimal incision. (B) Mucosal undermining was performed to expose the frontal process of the maxilla. (C) The incision extends posteriorly through the floor of the nose to the posterior portion of the inferior turbinate. (D) A straight osteotome was used to fracture the maxilla along the line of the original incision. (E) The entire medial wall of the maxilla, including the nasolacrimal duct and the inferior turbinate, were displaced medially. (F)The medial wall was returned to its original position and secured with two sutures. IT=inferior turbinate, $\mathrm{MT}=$ middle turbinate, $\mathrm{NF}=$ nasal floor, $\mathrm{NLD}=$ nasolacrimal duct, $\mathrm{NS}=$ nasal septum, $\mathrm{PW}=$ posterior wall.
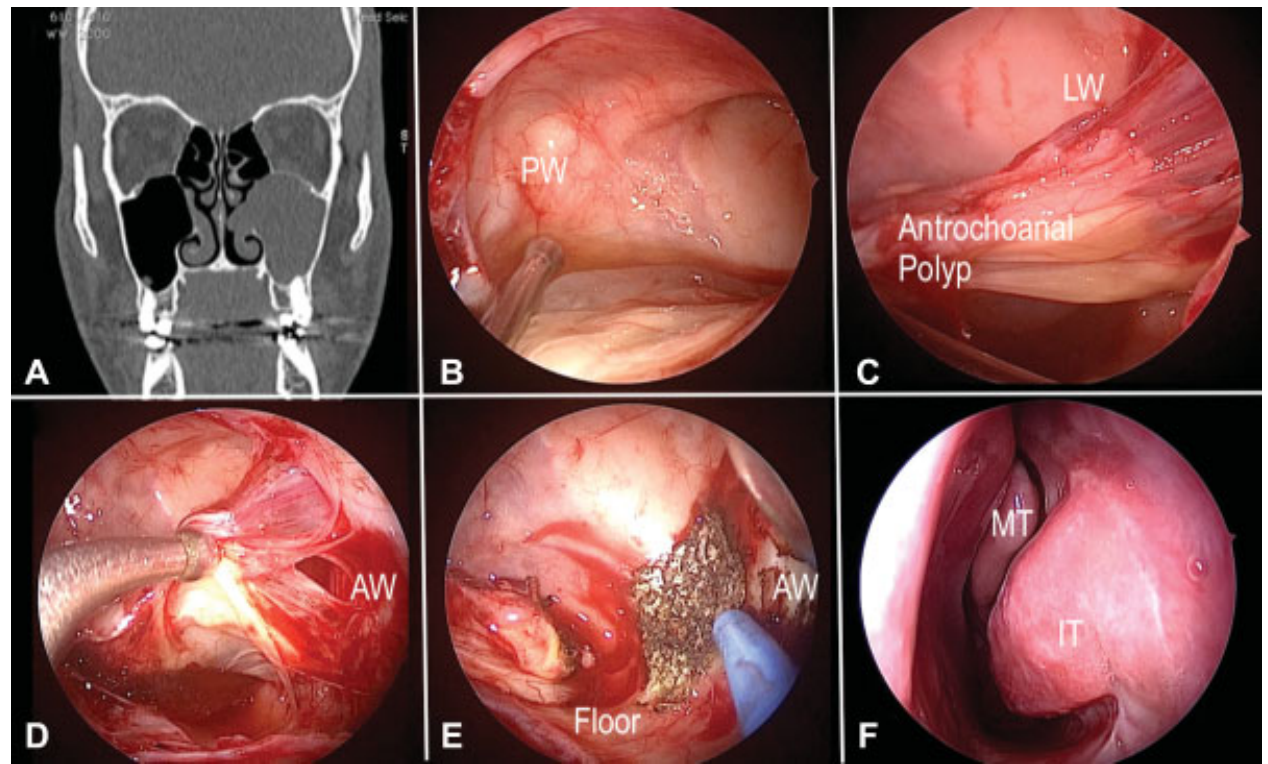

Fig. 2 Case 1, patient with antrochoanal polyp in the left nasal cavity and maxillary sinus (A) Coronal plane CT scan showing enlargement of the ostiomeatal complex and a mass lesion isodense (antrochoanal polyp) in the left maxillary sinus. (B) Endonasal approach of the left nasal fossa with a 45-degree scope. Exposure of the posterior wall of a left maxillary sinus after a wide middle antrostomy. (C) Endoscpic view with a 70-degree scope looking for the insertion of the antrochoanal polyp. (D, E) With a 30-degree scope, exposure of the insertion of the antrocoanal polyp, after medial maxillectomy and cauterization of the periosteum in the anterior wall of the maxillary sinus. (F) 2 months postoperative follow-up. $\mathrm{AW}=$ anterior wall, $\mathrm{IT}=$ inferior turbinate, $\mathrm{LW}=$ lateral wall, $\mathrm{MT}=$ middle turbinate, $\mathrm{MW}=$ medial wall, $\mathrm{NS}=\mathrm{nasal}$ septum, $\mathrm{PW}=$ posterior wall. 

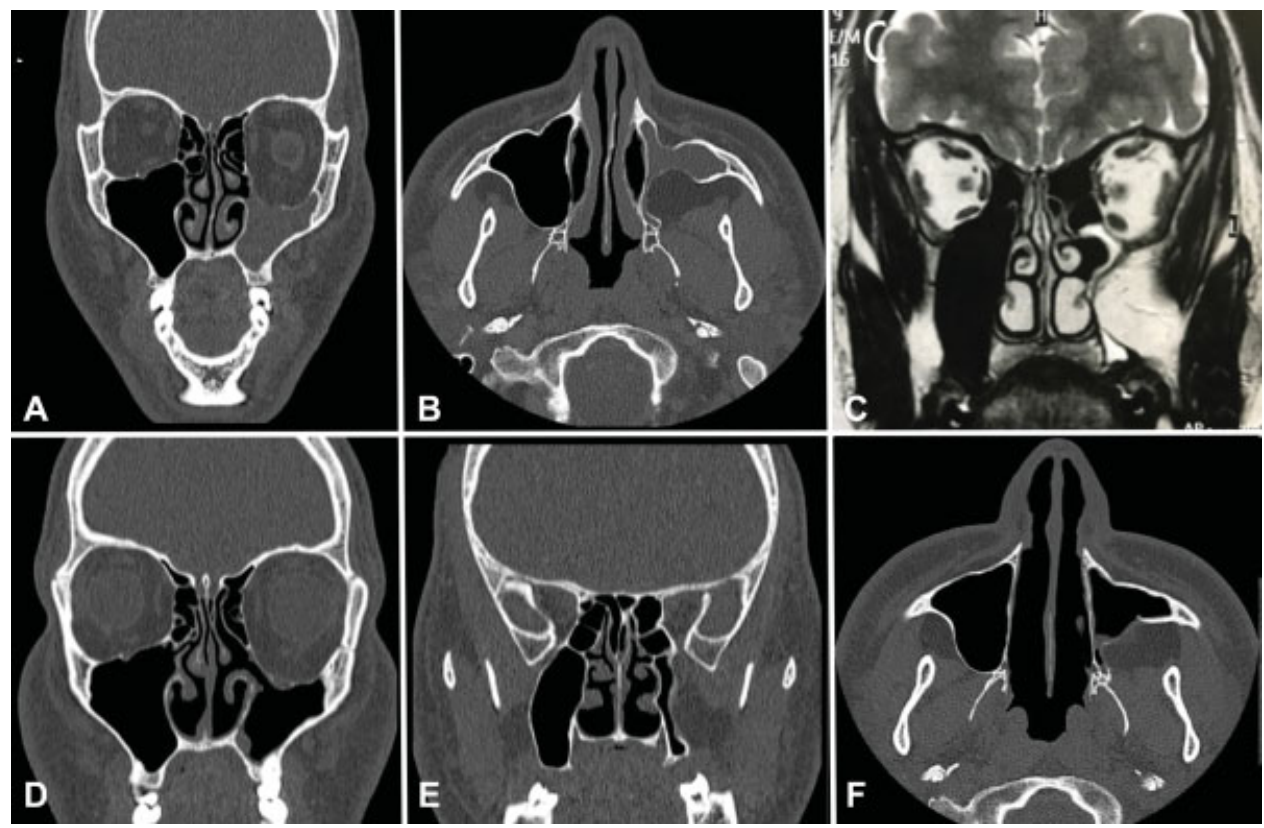

Fig. 3 Case 2, Silent sinus syndrome in the left maxillary sinus - Preoperative image exams (A-C); computed tomography (CT) scan in coronal plane (A), axial plane (B) and magnetic resonance imaging in T2 showing the compression of the lateral and posterior wall of the maxillary sinus by the infratemporal and pterygopalatine fossa. Postoperative CT scan, coronal plane (D, E) and axial plane (F). Observe the maxillary antrostomy and the bone integrity of the medial wall of the left maxillary sinus.

performed, which provided adequate access to the lesion, allowing complete excision, as well as drilling and cauterization of the periosteum (-Fig. 2B, C, D, E).

Histopathological analysis of the excised specimen confirmed the diagnosis of antrochoanal polyp.

The patient had an uneventful postoperative course, with improvement of nasal obstruction and hyposmia. $\mathrm{He}$ reported slight left-sided facial paresthesia, which resolved completely within 3 weeks. Nasal endoscopy showed no evidence of relapse at 1-year of follow-up (-Fig. 2F).

\section{Case 2}

In March 2018, a 30-year-old male presented with a chief complaint of chronic nasal congestion and progressive enophthalmos with a duration of several months.

Nasal endoscopy showed a left-sided septal deviation and discharge in the middle meatus. Computed tomography and magnetic resonance imaging (MRI) of the paranasal sinuses were consistent with silent sinus syndrome ( - Fig. 3A, B, C). There was significant enlargement of the posterior wall of the left maxillary sinus and of the fat pad of the ipsilateral infratemporal fossa.

Endoscopic endonasal surgery was performed. As in case 1 , a wide maxillary antrostomy was performed initially, which revealed significant downward displacement of the left orbital floor and a bulging posterior wall of the maxillary sinus. A left-sided REMM was performed to allow cauterization of the infratemporal fossa fat pad with bipolar diathermy.

The patient had an uneventful postoperative course, with improvement of his presenting complaints and no cosmetic or ocular sequelae. As in case 1 , he reported left-sided facial paresthesia, which improved spontaneously within 4 weeks.
A control CT of the paranasal sinuses performed at the 6month follow-up showed improved aeration of the left maxillary sinus and a $0.1-\mathrm{cm}$ reduction in displacement of the ipsilateral orbit ( - Fig. 3D, E, F). He continues to attend regular follow-up appointments for nasal endoscopy and measurement of orbital displacement.

\section{Case 3}

In july 2018, a 33-year-old male presented with a complaint of recurrent sinusitis, postnasal drip, and cacosmia secondary to a gunshot wound received 7 years previously. Three prior attempts at surgical treatment had been unsuccessful.

Nasal endoscopy revealed a purulent secretion in the middle meatus of the right nasal fossa and an ipsilateral oronasal fistula. The CT scan showed opacification of the right maxillary sinus with loss of bone discontinuity, as well as a bony defect in the floor of the nasal fossa.

Endoscopic endonasal surgery was performed, initially with a wide right antrostomy, followed by REMM to provide access and allow drilling of the right maxillary sinus floor. The procedure was completed by closure of the oronasal fistula with a mucosal flap raised from the floor and septum of the right nasal fossa.

The patient had an uneventful postoperative course, with improvement of his presenting complaints. He reported slight right-sided facial paresthesia, which improved spontaneously within 3 weeks. He remains asymptomatic at the periodic followup, with evidence of excellent healing on nasal endoscopy.

\section{Case 4}

A 50-year-old man with a history of postnasal discharge for 1 year. In September 2018, he still complained of chronic nasal obstruction. 


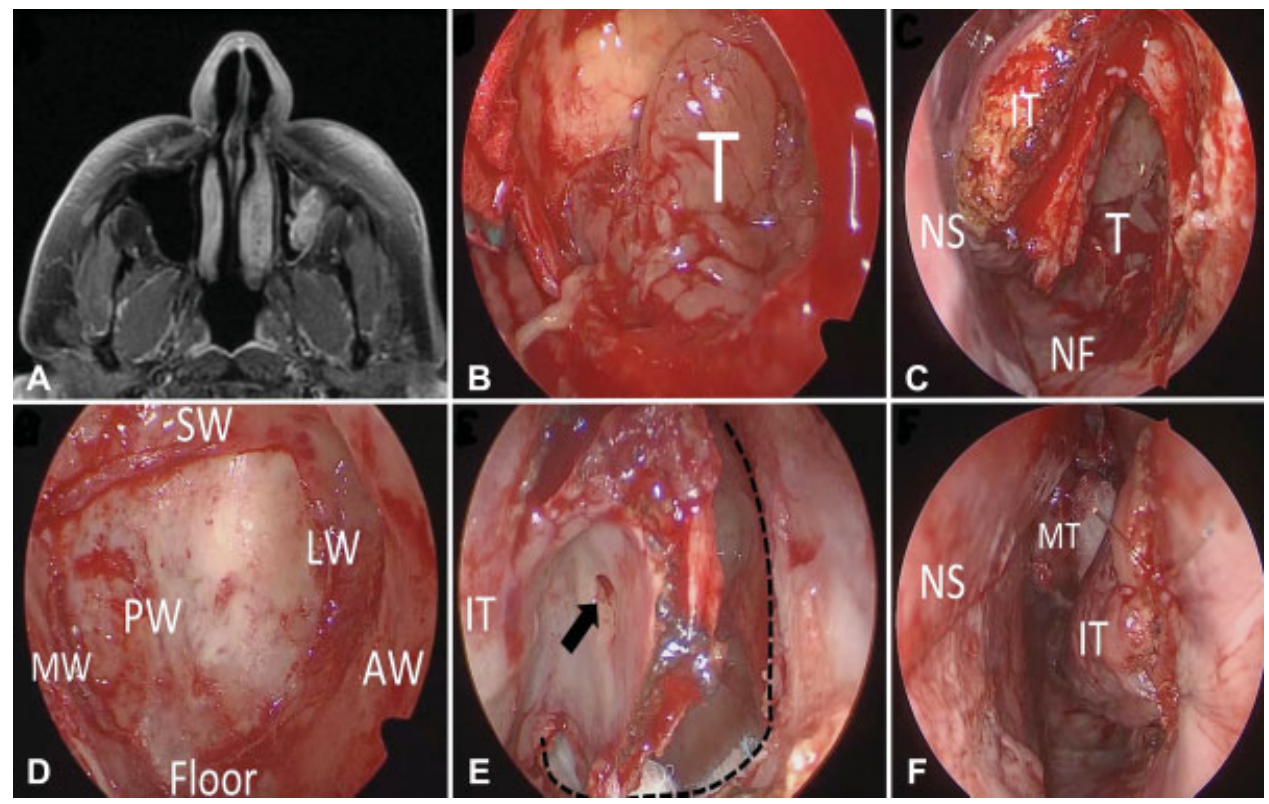

Fig. 4 Case 4, Inverted papilloma of the left maxillary sinus - (A) Magnetic resonance imaging showing the relation between the tumor and the anterior and lateral walls of the maxillary sinus. (B-F) Intraoperative photos from the left nasal cavity to show the REMM approach. (B) Endonasal view with a 45-degree scope, it is possible to observe the tumor occupying the left maxillary sinus after a wide middle antrostomy. (C) A 0-degree scope view of a REMM approach. (D) Endoscopic aspect with a 45-degree scope after tumor removal. (E) The limits of the osteotomy (dotted line) and the nasolacrimal duct ostium (arrow). (F) Suture of the incision anterior to the inferior turbinate. AW=anterior wall, $I T=$ inferior turbinate, $\mathrm{LW}=$ lateral wall, $\mathrm{MT}=$ middle turbinate, $\mathrm{MW}=$ medial wall, $\mathrm{NS}=$ nasal septum, $\mathrm{PW}=$ posterior wall, $\mathrm{SW}=$ superior wall of the maxillary sinus, $\mathrm{T}=$ tumor.

Nasal endoscopy showed a purulent discharge from the left middle meatus. The CT and MRI scans showed a solid lesion occupying the left maxillary sinus, with probable insertion in its lateral wall ( - Fig. 4A).

Initially, a large medium antrostomy was performed and an intraoperative frozen section biopsy indicated the diagnosis of an inverted papilloma. It was not possible to identify the insertion of the lesion in the maxillary sinus wall, even with a 70-degree endoscope view. Therefore, we chose to perform REMM for better access and visualization. The tumor was inserted in the transition of the lateral and anterior wall of the left maxillary sinus, being resected with subsequent periosteum drilling of this region with a diamond burr (-Fig. 4B, C, D, E, F).

The patient had a good evolution and was discharged on the same day. The anatomopathological study confirmed the diagnosis.

The two-month control nasal endoscopy shows optimal condition of the cavity, without nasal complications or patient complaints

\section{Discussion}

Open approaches to medial maxillectomy, such as the Caldwell-Luc procedure, lateral rhinotomy, and midfacial degloving, can provide wide access to the maxillary sinus. However, these techniques are associated with substantial morbidity. Complications, including epiphora, dacryocystitis, diplopia, mucocele, cerebrospinal fluid leak, epistaxis, and external scarring, are reported in $\sim 30 \%$ of the cases. ${ }^{4}$
The conventional endoscopic approach to medial maxillectomy consists of complete removal of the lateral nasal wall, including the inferior turbinate and the nasolacrimal duct. ${ }^{5}$ Although this approach provides satisfactory access to maxillary sinus lesions, excision of the medial wall of this sinus may result in dry nose, with massive postoperative crusting and mucociliary clearance disorders.

Subsequently, modified medial endoscopic maxillectomy techniques were developed in an attempt to preserve the sinonasal anatomy and physiology. Weber et al described a procedure that preserved the inferior turbinate but sacrificed the nasolacrimal duct, ${ }^{6}$ while Gras-Cabrerizo et al designed a technique which preserved both structures. ${ }^{7}$

Alternative endoscopic medial maxillectomy techniques, such as those described by Suzuki et al and by Maxfield et al, involve preservation of the inferior turbinate and nasolacrimal duct by displacing these structures medially to expose the bony medial wall of the maxillary sinus. A drill or osteotome is then used to remove the medial wall in the region of the inferior meatus. The original lateral nasal mucosa is replaced, or a mucous flap is raised from the nasal floor to cover the bone defect where access was obtained. ${ }^{8,9}$

The REMM technique provided wide access to maxillary sinus lesions just as well as the other endoscopic techniques described above. One of its advantages is maintenance of the sinonasal anatomy and physiology by preserving the inferior turbinate, the nasolacrimal duct, and the lateral wall of the nose (including its osteomucosal component). Unlike the other endoscopic approaches described previously, REMM does not necessitate removal of the bony component of the 


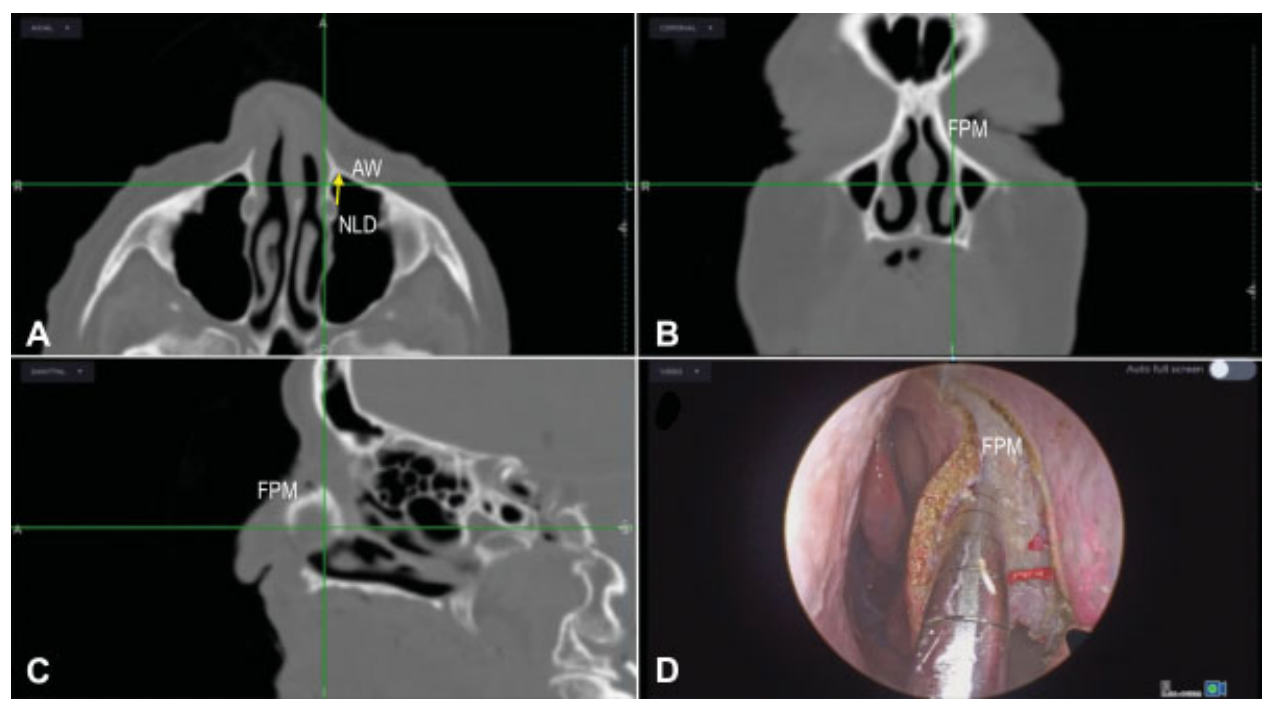

Fig. 5 Endonasal dissection with a 0-degree scope of the left nasal fossa with neuronavigation based on the computed tomography scan. Axial plane (A), coronal plane (B) and sagittal plane (C). It's possible to observe the distance between the nasolacrimal duct and the anterior wall of the maxillary sinus (A, yellow arrow). After electrocautery incision, correlation between the neuronavigation suction tip and the anatomy (D). AW= anterior wall, $\mathrm{FPM}=$ frontal process of maxilla, $\mathrm{NLD}=$ nasolacrimal duct.

medial wall of the maxillary sinus. Thus, there is minimal risk of postoperative fistula formation on the lateral wall of the nose. Furthermore, this technique minimizes postoperative crusting because the surgical wound bed is not exposed to inspired airflow.

Facial paresthesia is a frequent postoperative complication of all medial maxillectomy techniques, and is often persistent, which causes patient discomfort. ${ }^{10}$ In the present case series, this symptom resolved completely and spontaneously within 4 weeks of surgery.

One aspect that must be assessed during preoperative planning of REMM is maxillary sinus pneumatization and, consequently, the distance between the nasolacrimal duct and the pyriform sinus ( - Fig. 5 A, B, C, D). A poorly pneumatized maxillary sinus, with only a narrow space between the nasolacrimal duct and the pyriform aperture, will make osteotomy difficult and cause the procedure to be more challenging. Sieskiewicz et al analyzed the course of the nasolacrimal duct and assessed the distance between the nasolacrimal duct and the anterior wall of the maxillary sinus to determine the capability of performing a minimally invasive approach to the maxillary sinus. They demonstrated that in $\sim 30 \%$ of the cases, the lacrimal recess is so narrow that this type of approach might be difficult to perform without damaging the piriform aperture rim or the bony framework of the nasolacrimal duct. ${ }^{11}$ The same analysis needs to be done on patients who will be approached by REMM. This does not contraindicate this technique, but does require greater operator skill.

\section{Conclusion}

The REMM technique is an excellent surgical approach to benign conditions of the maxillary sinus. It has few limitations and appears to be associated with less morbidity than conventional techniques.
Conflict of Interests

The authors have no conflict of interests to declare.

\section{References}

1 Kasemsiri P, Prevedello DM, Otto BA, et al. Endoscopic endonasal technique: treatment of paranasal and anterior skull base malignancies. Rev Bras Otorrinolaringol (Engl Ed) 2013;79(06):760-779

2 Ali M, Nabil M, Safa N, et al. Diagnosis and management of benign tumors of nasal and paranasal cavities: 31 cases. Egypt J Otolaryngol 2015;31(01):4

3 Tanna N, Edwards JD, Aghdam H, Sadeghi N. Transnasal endoscopic medial maxillectomy as the initial oncologic approach to sinonasal neoplasms: the anatomic basis. Arch Otolaryngol Head Neck Surg 2007;133(11):1139-1142

4 Maxfield AZ, Chen TT, Scopel TF, et al. Transnasal endoscopic medial maxillary sinus wall transposition with preservation of structures. Laryngoscope 2016;126(07):1504-1509

5 Nakayama T, Asaka D, Okushi T, Yoshikawa M, Moriyama H, Otori N. Endoscopic medial maxillectomy with preservation of inferior turbinate and nasolacrimal duct. Am J Rhinol Allergy 2012;26 (05):405-408

6 Weber RK, Werner JA, Hildebrand T. Endonasal endoscopic medial maxillectomy with preservation of the inferior turbinate. Am J Rhinol Allergy 2010;24:132-135

7 Gras-Cabrerizo JR, Massegur-Solench H, Pujol-Olmo A, MontserratGili JR, Adema-Alcover JM, Zarraonandia-Andraca I. Endoscopic medial maxillectomy with preservation of inferior turbinate. how do we do it? Eur Arch Otorhinolaryngol 2011;268:389-392

8 Vrabec DP. The inverted Schneiderian papilloma: a 25-year study. Laryngoscope 1994;104(5 Pt 1):582-605

9 Sadeghi N, Al-Dhahri S, Manoukian JJ. Transnasal endoscopic medial maxillectomy for inverting papilloma. Laryngoscope 2003;113(04):749-753

10 Giao M, Lameira A, Lopes P, Fino R, Montalvão P, Magalhães M. Carcinoma Adenoide Cístico das fossas nasais e seios perinasais Revisão retrospectiva de 20 anos. Rev Portuguesa Otorrinolaringol Cirurgia Cabeça Pescoço 2018;55(03):163-166

11 Sieskiewicz A, Buczko K, Janica J, et al. Minimally invasive medial maxillectomy and the position of nasolacrimal duct: the CT study. Eur Arch Otorhinolaryngol 2017;274(03):1515-1519 